\title{
DEVELOPMENT OF AN INSTRUMENT FOR NON-DESTRUCTIVE IDENTIFICATION OF UNEXPLODED ORDNANCE USING TAGGED NEUTRONS - A PROOF OF CONCEPT STUDY
}

\author{
S. Mitra and I. Dioszegi
}

For presentation at the IEEE Nuclear Science Symposium and Medical Imaging Conference Valencia, Spain October 23-29, 2011

July 2011

\section{Environmental Sciences Department/Energy Research and Technology Division Brookhaven National Laboratory \\ P.O. Box 5000 \\ Upton, NY 11973-5000 \\ www.bnl.gov}

Notice: This manuscript has been authored by employees of Brookhaven Science Associates, LLC under Contract No. DE-AC0298CH10886 with the U.S. Department of Energy. The publisher by accepting the manuscript for publication acknowledges that the United States Government retains a non-exclusive, paid-up, irrevocable, world-wide license to publish or reproduce the published form of this manuscript, or allow others to do so, for United States Government purposes. 


\title{
Development of an instrument for non-destructive identification of Unexploded Ordnance using tagged neutrons - a proof of concept study
}

\author{
S. Mitra and I. Dioszegi
}

\section{INTRODUCTION}

Range clearance operations at munitions testing grounds must discriminate Unexploded Ordnance (UXO) from clutter items and distinguish UXO filled with High Explosives (HE) from those with inert fillers. Non-destructive technologies are thus necessary for the cost-effective disposal of UXO during remediation of such sites. The only technique showing promise so far for the non-destructive elemental characterization of UXO fillers utilizes neutron interactions with the material to detect carbon $(\mathrm{C})$, nitrogen $(\mathrm{N})$ and oxygen (O) which have unique ratios in HE. However, several unresolved issues hinder the wide application of this potentially very suitable technique. The most important one is that neutrons interact with all surrounding matter in addition to the interrogated material, leading to a very high gamma-ray background in the detector. Systems requiring bulky shielding and having poor signal-tonoise ratios (SNRs) for measuring elements are unsuitable for field deployment.

The inadequacies of conventional neutron interrogation methods are overcome by using the taggedneutron approach, and the availability of compact sealed neutron generators exploiting this technique $[1,2]$ offers field deployment of non-intrusive measurement systems for detecting threat materials, like explosives and drugs. By accelerating deuterium ions into a tritium target, the subsequent fusion reaction generates nearly back-toback emissions of neutrons and alpha particles of energy 14.1 and $3.5 \mathrm{MeV}$ respectively. A position-sensitive detector recognizes the associated alpha particle, thus furnishing the direction of the neutron. The tagged neutrons interact with the nuclei of the interrogated object, producing element-specific prompt gamma-rays that the gamma detectors recognize. Measuring the delay between the detections of the alpha particle and the gamma-ray determines where the reaction occurred along the axis of the neutron beam (14.1 MeV neutrons travel at $5 \mathrm{~cm} /$ nanosecond, while gamma rays cover 30

This work was supported by the U.S. Department of Defense under it's Strategic Environmental Research and Development Program (SERDP), Project\# MR 1769. S Mitra is with the Environmental Sciences Department (emailsmitra@bnl.gov) and I. Dioszegi is with the Nonproliferation and National Security Department (email dioszegi@bnl.gov) of Brookhaven National Laboratory, Upton, NY 11973 USA $\mathrm{cm} /$ nanosecond). The main advantage of the technique is its ability to simultaneously provide $2 \mathrm{D}$ and $3 \mathrm{D}$ imaging of objects and their elemental composition. This work reports on the efficacy of using $14 \mathrm{MeV}$ neutrons tagged by the associated particle neutron time-of-flight technique (APnTOF) to extract neutron induced characteristic gamma-rays from an object-of-interest with high SNR and without interference from nearby clutter.

\section{METHODS}

A small portable API120 neutron generator(NG) was operated at a continuous output of $\sim 3 \times 10^{7} \mathrm{n} / \mathrm{s}$ while the gamma-rays were detected using a $12.7 \times 12.7 \mathrm{~cm}$ diameter $\mathrm{NaI}(\mathrm{Tl})$ detector. The alpha particle detection system comprised a $5 \mathrm{~cm}$ diameter fast Amperex XP2020 PMT that was mated with the $6.5 \mathrm{~cm}$ fiber-optic face-plate of the neutron generator's alpha detector. Standard NIM electronics was used for the alpha-gamma coincidence spectroscopy. A graphite cube of dimension $15.2 \mathrm{~cm}$ (mass $8 \mathrm{~kg}$ ) was used as the object-of-interest ("UXO") while a half-gallon bottle of water and a $5 \mathrm{~mm}$ thick iron slab were used as clutter items. Three cases were investigated to extract carbon signals from the graphite without interference from the oxygen signals from water: In case 1 (no overlapping clutter), the bottle of water was outside the defined neutron cone, cases 2 and 3 (overlapping clutter) progressively introduced the bottle of water and iron slab between the neutron beam and the graphite sample. For all cases the graphite block was at a distance of $47 \mathrm{~cm}$ along the axis of the neutron beam and the bottle of water was at a radial distance of $27 \mathrm{~cm}$ from the NG target. In a separate series of experiments, time correlated gamma spectra of the graphite block and a one gallon bottle of water were independently determined when located in the neutron cone at a distance of $27 \mathrm{~cm}$ from the target. The SNR are compared with the respective gamma-ray spectra when no coincidence time-gate was applied.

\section{RESULTS AND DISCUSSION}

Fig. 1 shows the time dependence of $\alpha-\gamma$ coincidences for the object positioned at a distance of $27 \mathrm{~cm}$ from the NG target. The time correlated gamma-ray spectra of carbon and oxygen were obtained with a $10 \mathrm{~ns}$ time 
window and are shown in Figs. 2 and 3 respectively. The SNR at the peak energies of $4.43(\mathrm{C})$ and $6.13 \mathrm{MeV}(\mathrm{O})$ were found to be 1.3 when spectra were acquired without the time coincidence mode. The SNRs were vastly improved; 22 and 10 for $\mathrm{C}$ and $\mathrm{O}$ respectively in the coincidence mode.

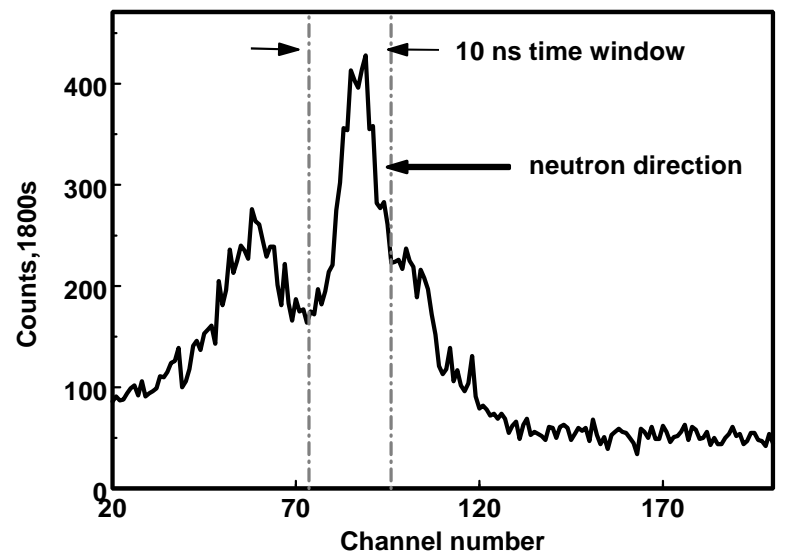

Fig.1 Time distribution of $\alpha-\gamma$ coincidences for an object $27 \mathrm{~cm}$ from the NG target.

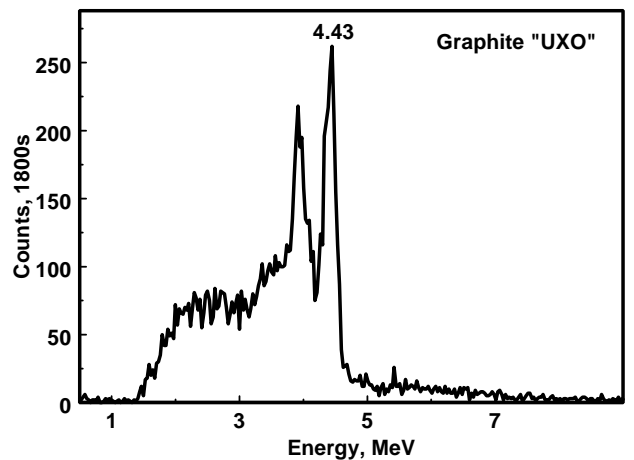

Fig.2 Time correlated $\gamma$-ray spectrum of carbon.

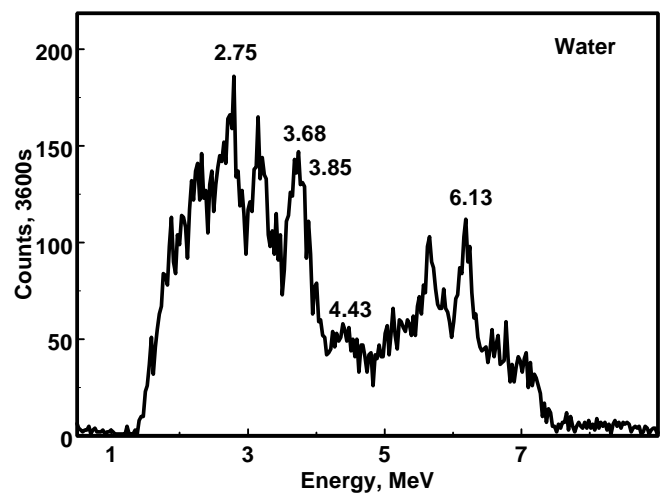

Fig.3 Time correlated $\gamma$-ray spectrum of oxygen.

Fig. 4 shows the time spectra of the objects when present separately in the beam and when present simultaneously. A suitable time window, $5.6 \mathrm{~ns}$ wide was set for the "UXO" and it's time correlated gammaray spectrum was extracted for the three cases. Fig.5 shows the inelastic gamma-rays of energy $4.43 \mathrm{MeV}$ from carbon ("UXO") under the different clutter conditions. It can be seen that the oxygen signals from water are absent in all the three cases. The lower energy signals below $3 \mathrm{MeV}$ for cases 2 and 3 are a result of additional time correlated neutron scattering effects because of the near-by overlapping clutter.

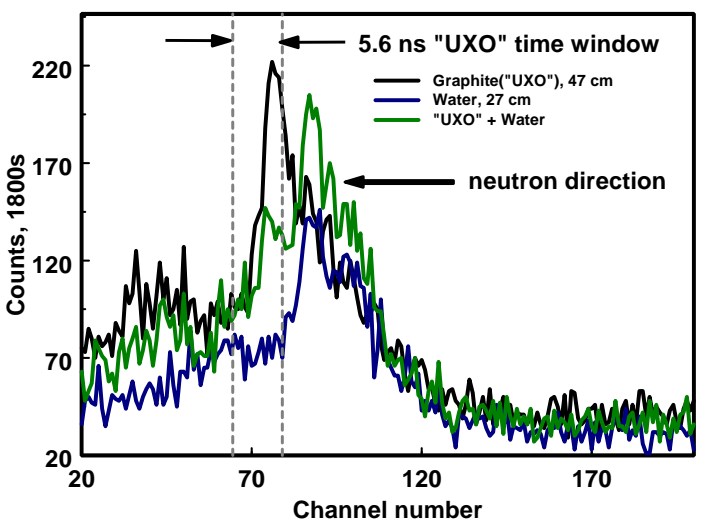

Fig.4 Time spectra of objects present separately and under clutter condition in the defined neutron cone.

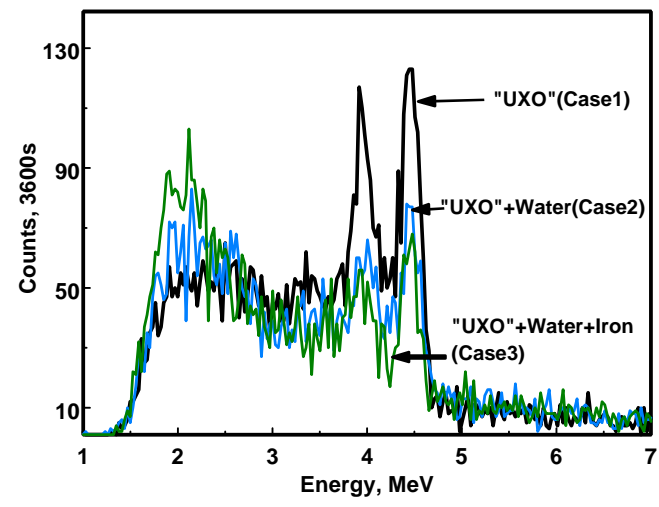

Fig.5 Time correlated $\gamma$-ray spectra of the "UXO" under nonoverlapping (Case1) and overlapping (Case2 and 3) clutter conditions using a $5.6 \mathrm{~ns}$ time window.

\section{CONCLUSION}

In contrast to conventional neutron interrogation techniques, the results clearly demonstrated the efficacy of the neutron time-of flight technique for obtaining gamma-ray signals from an object-of-interest with (1) high SNR and (2) without interference from signals of near-by clutter.

\section{REFERENCES}

1. S. Mitra, J.E. Wolff, R. Garrett, C.W. Peters, Application of the associated particle technique for the whole-body measurement of protein, fat and water by $14 \mathrm{MeV}$ neutron activation analysis- a feasibility study. Phys Med Biol 40(6): 1045-1055, 1995.

2. D.L. Chichester, M. Lemchak, J.D. Simpson, The API 120: A portable neutron generator for the associated particle technique. Nucl. Inst. Methods Phys Res Sect B 241(1-4):753-758, 2005. 